\title{
Long-term Outcome of Ventricular Tachycardia Ablation in Patients who did not Undergo Programmed Electrical Stimulation after Ablation
}

\author{
Takeshi Kitamura ${ }^{1}$, Seiji Fukamizu ${ }^{1}$, Tomoyuki Arai ${ }^{1}$, Kohei Kawajiri ${ }^{1}$, Sho Tanabe ${ }^{1}$, \\ Sayuri Tokioka ${ }^{1}$, Dai Inagagaki ${ }^{1}$, and Rintaro Hojo ${ }^{1}$ \\ ${ }^{1}$ Tokyo Metropolitan Hiroo Hospital
}

May 18, 2020

\begin{abstract}
Background: Ventricular arrhythmia inducibility is one of the ideal endpoints of ventricular tachycardia(VT) ablation. However, it may be challenging to implement programmed electrical stimulation (PES) at the end of the procedure under several circumstances. The long-term outcome of patients who did not undergo PES after VT ablation remains largely unknown. Purpose: To investigate the details and long-term outcome of VT ablation in patients who did not undergo PES at the end of the ablation procedure. Methods: Among $184 \mathrm{VT}$ ablation procedures in patients with structural heart disease who underwent VT ablation using an irrigated catheter, we enrolled those who did not undergo PES after VT ablation. VT ablation strategy involved targeting induced VT plus pacemap-guided substrate ablation if inducible. If VT was not inducible, substrate-based ablation was performed. The primary endpoint was VT recurrence. Results: In 58 procedures, post-ablation VT inducibility was not assessed. The causes were non-inducibility of sustained VT before ablation(27/58, 46.6\%), long procedure time(27.6\%, mean $392 \mathrm{~min}$ ), complications(10.3\%), intolerant hemodynamic state(10.3\%), and inaccessible or unsafe target(6.9\%). With regard to the primary endpoint, 23 recurrences(39.7\%) were observed during a mean follow-up period of 2.5 years. Patients with non-inducibility before ablation showed less VT recurrences $(4 / 27,14.8 \%)$ during follow-up than patients with other causes of untested PES after ablation $(19 / 31,61.2 \%)($ Log-rank<0.001). Conclusions: VT recurrence was not observed in approximately $60 \%$ of the patients who did not undergo PES at the end of the ablation procedure. PES after VT ablation may be not needed among patients with pre-ablation non-inducibility.
\end{abstract}

\section{Introduction}

Several studies have demonstrated the efficacy of radiofrequency catheter ablation as an adjunctive therapy for selected patients with structural heart disease (SHD) and drug-resistant ventricular tachycardia (VT) $)^{1,2}$. Different ablation strategies have been proposed, and their outcomes varied across clinical trials ${ }^{3-9}$. Generally, the ideal endpoint of VT ablation in patients with SHD is non-inducibility of VT at the end of the ablation procedure ${ }^{10-12}$. Several retrospective comparative studies and meta-analysis have demonstrated that patients in whom VT cannot be induced at the conclusion of an ablation have a favorable outcome ${ }^{10-15}$. However, these studies have been limited because they lack data of patients who did not undergo programmed electrical stimulation (PES) at the end of the ablation procedure and data on the long-term follow-up of those patients. To answer this important clinical real-world question, we aimed to evaluate details and outcomes of patients who did not undergo PES at the end of the ablation procedure through our single-center experience.

\section{Methods}

Study population 
A total of 183 ablation procedures in patients with SHD who underwent catheter ablation for VT by using an irrigated ablation catheter between November 2009 and December 2018 were retrospectively searched. This retrospective observational analysis evaluated VT ablation procedures in patients with SHD in whom PES was not performed at the end of the VT ablation procedure. The study was approved by Institutional Review Boards at Tokyo Metropolitan Hiroo Hospital, all patients provided written informed consent.

VT ablation protocol

Our electrophysiological study protocol was already described in our previous work ${ }^{16}$. Briefly, electrophysiological study included the delivery of 1-3 extrastimuli during pacing at two basic cycle lengths (400 and $600 \mathrm{~ms}$ ) from two right ventricular (RV) sites [RV apex (RVA) and RV outflow tract] and burst pacing. A target VT was defined as an induced sustained monomorphic VT or a clinically documented VT. Catheter ablation was performed using an irrigated ablation catheter (ThermoCool or ThermoCool SF or ThermoCool STSF; Biosense Webster, CA, or Coolpath or Tacticath; Abbott, MN) via a transvenous and/or retrograde transaortic approach and/or percutaneous epicardial approach. For patients with inducible hemodynamically tolerated monomorphic VT, CA was performed at sites with mid-diastolic potential, where pacing entrained the VT with concealed fusion, a post-pacing interval within $30 \mathrm{~ms}$ of the VT cycle length (VTCL), and a stimulus-to-QRS interval of $70 \%$ of the VTCL with or without using a three-dimensional (3D) electroanatomical (EA) mapping system (CARTO, Biosense Webster or Ensite, Abbott, MN). In cases in which the 3D EA mapping system had been used, bipolar endocardial voltage mapping of the left ventricle (LV) was performed during sinus or RV pacing rhythm. The low-voltage area was defined by voltage criteria [?]1.5 $\mathrm{mV}^{7,17-19}$. Then, substrate modification was additionally performed based on the abnormal electrograms: fragmented electrograms and delayed electrograms with the operator's discretion.

For patients with inducible hemodynamically intolerant monomorphic VT, exit sites and VT channels had been identified and ablated on the basis of the pace mapping. Then, substrate modification was additionally performed based on the abnormal electrograms with the operator's discretion.

When VT was not inducible at the beginning of the procedure, substrate modification based on the abnormal electrograms was implemented.

PES was repeated after VT ablation. Successful ablation was defined as non-inducibility of the target monomorphic VT at the end of the procedure. However, when VT was not inducible at the beginning of the procedure, PES after RF delivery was not performed.

Furthermore, among the procedures without PES at the end of the procedure, we evaluated the number of procedures without sufficient RF delivery because of the reasons of untested PES.

Follow-up

Patients were followed every 3 months thereafter. Implantable cardioverter defibrillators were evaluated at each visit, and arrhythmia logs were retrieved. The primary endpoint was a recurrent VT. In addition, we evaluated VT recurrence rate after VT ablation among the total procedures divided into four groups: 1) a target VT was not inducible at the end of the procedure, 2) a target VT was still inducible at the end of the procedure, 3) PES was not implemented because of non-inducibility before RF delivery, 4) PES was not implemented because of other causes.

\section{Statistical analysis}

Continuous variables are presented as mean \pm standard deviation or median \pm interquartile range (IQR, 2575th percentile), depending on the normality of distribution. Categorical variables are reported using numbers and percentages. Event-free survival curves were constructed using the Kaplan-Meier method and compared using the log-rank test. We compared the prevalence of VT recurrence at 2 years between two groups using the Wilcoxon rank-sum test. Event-free survival curves among four groups in the total population were also constructed using the Kaplan-Meier method and compared using the log-rank test. Bonferroni test for posthoc analysis for the intergroup comparison of each pair of groups. This post-hoc test sets the significance cut 
off at $0.05 / 4(=0.0125)$. All reported P-values are two-sided, with a P-value of $<0.05$ considered to indicate statistical significance. Statistical analyses were performed using the Statistical Package for Social Studies version 25.0 (IBM Corp., Armonk, NY, USA).

\section{Results}

Patient characteristics

Among the total of 183 ablation procedures, we enrolled a total of 58 procedures (31.7\%: 58/183), where PES after VT ablation was not performed. Patient characteristics are shown in Table 1 . The mean age was 67 \pm 10 years, with a mean left ventricular ejection fraction of $35 \pm 11 \%$. Among the rest of procedures (125), when considering inducibility of a target VT at the end of the procedure, target VTs were not inducible at the end of the procedure in 116 procedures (92.8\%: 116/125) while target VTs were still inducible at the end of the procedure in nine procedures $(7.2 \%: 9 / 125)$.

Procedural details

Peri-procedural details are shown in Table 2. In all procedures, a 3D mapping system and an irrigated ablation catheter were utilized during VT ablation. A force-sensing ablation catheter, a multipolar mapping catheter, and an intracardiac echocardiography incorporated with the 3D mapping system were used in $44.8 \%, 69.0 \%$, and $64.0 \%$ of the procedures, respectively.

The rationale of avoiding PES after RF delivery is shown inTable 3 . The most frequent cause was noninducibility of VT before RF delivery (46.6\%). Following this, procedure time of $>6$ hours (average $392 \pm 59$ min), complications ( 2 cardiac tamponade, 2 transient atrioventricular block, 1 cardiac arrest, 1 air emboli in right coronary artery), intolerant hemodynamic state, and an inaccessible/unsafe target (1 His area, 1 right bundle area, and 1 epicardial origin) were observed. With regard to the prevalence of procedure suspension before sufficient RF delivery, required RF delivery was applied in all procedures with non-inducibility of VT before RF delivery whilst more than $80 \%$ of procedures were suspended before sufficient RF delivery in procedures with complications or inaccessible/unsafe target (Table 3 ).

Primary endpoint

A total of 23 VT recurrences (39.6\%) occurred during a mean follow-up period of 2.5 years. Patients who did not undergo PES after RF delivery due to non-inducibility before RF delivery, showed significantly higher VT-free survival rate during the follow-up period than those who did not undergo PES because of other causes (Log-rank < 0.001) (Figure 1 ). The prevalence of VT recurrence-free patients at 2 years after VT ablation was significantly higher in patients with non-inducibility before RF delivery than those with other causes (92\% vs. $36 \%$; $\mathrm{P}<0.001)$.

Additionally, we evaluated VT recurrence rate after VT ablation among the total procedures divided into four groups: 1) where a target VT was not inducible at the end of the procedure, 2) where a target VT was still inducible at the end of the procedure, 3) where PES was not implemented because of non-inducibility before RF delivery, 4) where PES was not implemented because of other causes (Supplementary Figure 1 ). Additional ad-hoc analysis of each pair of groups were also noted in the figure.

\section{Discussion}

Our main findings are as follows: (1) approximately $60 \%$ of the patients had no VT recurrences during a mean follow-up period of 2.5 years when PES was not implemented after VT ablation due to variable causes. (2) The prevalence of VT recurrence-free rate at 2 years after VT ablation was significantly higher in patients with non-inducibility before RF delivery than in those with other causes.

Non-inducibility after VT ablation is known as one of the ideal endpoints ${ }^{10}$. However, PES after VT ablation can be occasionally harmful or omitted under several circumstances ${ }^{20}$. Several retrospective studies evaluated the effect of non-inducibility after VT ablation and showed that $8 \%^{-16} \%^{12,21}$ of the patients who underwent VT ablation did not undergo PES after VT ablation. However, to our knowledge, no studies have 
described the rationales of these situations that are not infrequent to encounter in real-world clinical situation. Although the variety and frequency of the causes depend on the institutional or operator's discretion, our study demonstrated that non-inducibility before ablation was the leading cause (reportedly, VTs are not inducible before ablation among $37 \%$ of VT ablation patients ${ }^{22}$ ). This was followed by long procedure time, complications, intolerant hemodynamic state, and inaccessible or unsafe targets.

With respect to the long-term follow-up, most of the studies evaluated VT recurrences of patients who did not undergo PES after VT ablation combined with patients who were still inducible after VT ablation ${ }^{12,14,23}$. No studies have investigated the VT recurrence in an independent untested group. Our study first demonstrated the long-term follow-up of this untested group that was approximately $60 \%$ VT-free during a mean follow-up of 2.5 years. Furthermore, the prevalence of VT recurrence at 2 years after VT ablation was significantly lower in patients with non-inducibility before RF delivery than those with other causes. This finding seems logically acceptable because the former was considered less arrhythmogenic while the latter was considered an incomplete procedure as shown by the prevalence of suspension before sufficient RF delivery in Table 3. In addition, the long-term follow-up of untested group with non-inducibility before RF delivery shows as favorable result as the procedures with non-inducibility of target VTs (Supplementary Figure 1 ). Therefore, considering this finding with approximately $90 \%$ of VT-free survival rate at 2 years, PES after VT ablation might be not an ideal endpoint in patients with non-inducibility before RF delivery. Furthermore, importantly this etiology (so called untested PES at the end of ablation) was heterogeneous, which may require careful post-procedural follow-up based on the rationale of untested PES.

\section{Study limitations}

Our study has several limitations. First, it was a descriptive retrospective single-center analysis and consisted of a relatively small population who were strictly selected and eligible for VT ablation. Second, the implementation of PES after VT ablation in patients who were not inducible before VT ablation depends on the institutional or operator's discretion. Therefore, generalizability is unknown although this study's finding provided important real-world information that has never been described so far. Further investigations to evaluate the generalizability in a multicenter-manner are needed.

\section{Conclusions}

VT recurrence was not observed in approximately $60 \%$ of the patients who did not undergo PES at the end of the ablation procedure. PES after VT ablation may not be needed among patients with non-inducibility before ablation.

\section{Acknowledgements}

None.

\section{Disclosure}

None.

\section{References}

1. Kuck K-HH, Schaumann A, Eckardt L, et al. Catheter ablation of stable ventricular tachycardia before defibrillator implantation in patients with coronary heart disease (VTACH): a multicentre randomised controlled trial. Lancet . 2010;375(9708):31-40. doi:10.1016/S0140-6736(09)61755-4

2. Reddy VY, Reynolds MR, Neuzil P, et al. Prophylactic catheter ablation for the prevention of defibrillator therapy. $N$ Engl J Med . 2007;357(26):2657-2665. doi:10.1056/NEJMp1002530

3. Jaïs P, Maury P, Khairy P, et al. Elimination of local abnormal ventricular activities : A new end point for substrate modification in patients with scar-related ventricular tachycardia. Circulation . 2012;125(18):21842196. doi:10.1161/CIRCULATIONAHA.111.043216 
4. Briceno DF, Romero J, Villablanca PA, et al. Long-term outcomes of different ablation strategies for ventricular tachycardia in patients with structural heart disease: systematic review and meta-analysis. $E P$ Eur . 2017:1-12. doi:10.1093/europace/eux109

5. Di Biase L, Santangeli P, Burkhardt DJ, et al. Endo-epicardial homogenization of the scar versus limited substrate ablation for the treatment of electrical storms in patients with ischemic cardiomyopathy. $J$ Am Coll Cardiol . 2012;60(2):132-141. doi:10.1016/j.jacc.2012.03.044

6. Aziz Z, Tung R. Novel Mapping Strategies for Ventricular Tachycardia Ablation. Curr Treat Options Cardiovasc Med . 2018;20(4). doi:10.1007/s11936-018-0615-1

7. Marchlinski FE, Callans DJ, Gottlieb CD, Zado ES. Linear Ablation Lesions for Control of Unmappable Ventricular Tachycardia in Patients With Ischemic and Nonischemic Cardiomyopathy Francis E. Marchlinski, David J. Callans, Charles D. Gottlieb and Erica Zado Circulation 2000;101;1288-1296. Circulation . 2000;101:1288-1296.

8. Berruezo A, Fernandez-Armenta J, Andreu D, et al. Scar dechanneling.Circ Arrhythmia Electrophysiol . 2015;8(2):326-336. doi:10.1161/CIRCEP.114.002386

9. Tzou WS, Frankel DS, Hegeman T, et al. Core isolation of critical arrhythmia elements for treatment of multiple scar-based ventricular tachycardias. Circ Arrhythmia Electrophysiol . 2015;8(2):353-361. doi:10.1161/CIRCEP.114.002310

10. Cronin EM, Bogun FM, Maury P, et al. 2019 HRS/EHRA/APHRS/LAHRS Expert Consensus Statement on Catheter Ablation of Ventricular Arrhythmias . Vol 35. Wiley-Blackwell; 2019:323-484. http://www.ncbi.nlm.nih.gov/pubmed/31293696. Accessed June 6, 2019.

11. Hu J, Zeng S, Zhou Q, et al. Can ventricular tachycardia non-inducibility after ablation predict reduced ventricular tachycardia recurrence and mortality in patients with non-ischemic cardiomyopathy? A meta-analysis of twenty-four observational studies. Int J Cardiol . 2016;222:689-695. doi:10.1016/j.ijcard.2016.07.200

12. Essebag V, Joza J, Nery PB, et al. Prognostic Value of Noninducibility on Outcomes of Ventricular Tachycardia Ablation: A VANISH Substudy. JACC Clin Electrophysiol . 2018;4(7):911-919. doi:10.1016/j.jacep.2018.03.013

13. Sauer WH, Zado E, Gerstenfeld EP, Marchlinski FE, Callans DJ. Incidence and predictors of mortality following ablation of ventricular tachycardia in patients with an implantable cardioverter-defibrillator.Hear Rhythm . 2010;7(1):9-14. doi:10.1016/j.hrthm.2009.09.014

14. Ghanbari H, Baser K, Yokokawa M, et al. Noninducibility in postinfarction ventricular tachycardia as an end point for ventricular tachycardia ablation and its effects on outcomes a meta-analysis. Circ Arrhythmia Electrophysiol . 2014;7(4):677-683. doi:10.1161/CIRCEP.113.001404

15. Carbucicchio C, Santamaria M, Trevisi N, et al. Catheter ablation for the treatment of electrical storm in patients with implantable cardioverter-defibrillators : Short-and long-term outcomes in a prospective single-center study. Circulation . 2008;117(4):462-469. doi:10.1161/CIRCULATIONAHA.106.686534

16. Hayashi T, Fukamizu S, Hojo R, et al. Prophylactic catheter ablation for induced monomorphic ventricular tachycardia in patients with implantable cardioverter defibrillators as primary prevention. EP Eur . 2013;15(10):1507-1515. doi:10.1093/europace/eut050

17. Soejima K, Stevenson WG, Sapp JL, Selwyn AP, Couper G, Epstein LM. Endocardial and epicardial radiofrequency ablation of ventricular tachycardia associated with dilated cardiomyopathy: The importance of low-voltage scars. J Am Coll Cardiol . 2004;43(10):1834-1842. doi:10.1016/j.jacc.2004.01.029

18. Soejima K, Stevenson WG, Maisel WH, Sapp JL, Epstein LM. Electrically unexcitable scar mapping based on pacing threshold for identification of the reentry circuit isthmus: Feasibility for guiding ventricular 
tachycardia ablation. Circulation . 2002;106(13):1678-1683. doi:10.1161/01.CIR.0000030187.39852.A7

19. Santangeli P, Marchlinski FE. Substrate mapping for unstable ventricular tachycardia. Hear Rhythm . 2016;13(2):569-583. doi:10.1016/j.hrthm.2015.09.023

20. Callans DJ. Can we eliminate noninducibility by programmed stimulation as an endpoint for ventricular tachycardia ablation in patients with structural heart disease? J Cardiovasc Electrophysiol . 2012;23(6):628630. doi:10.1111/j.1540-8167.2011.02274.x

21. Stevenson WG, Wilber DJ, Natale A, et al. Irrigated radiofrequency catheter ablation guided by electroanatomic mapping for recurrent ventricular tachycardia after myocardial infarction: the multicenter thermocool ventricular tachycardia ablation trial. Circulation . 2008;118(25):2773-2782. doi:10.1161/CIRCULATIONAHA.108.788604

22. Arenal A, Glez-Torrecilla E, Ortiz M, et al. Ablation of electrograms with an isolated, delayed component as treatment of unmappable monomorphic ventricular tachycardias in patients with structural heart disease. J Am Coll Cardiol . 2003;41(1):81-92. doi:10.1016/S0735-1097(02)02623-2

23. Yokokawa M, Kim HM, Baser K, et al. Predictive value of programmed ventricular stimulation after catheter ablation of post-infarction ventricular tachycardia. J Am Coll Cardiol . 2015;65(18):1954-1959. doi:10.1016/j.jacc.2015.02.058

Table 1. Procedures characteristics

\begin{tabular}{ll}
\hline & $\mathrm{N}=58$ \\
\hline Age, (y) & $67 \pm 10$ \\
Sex, male/female, n/n & $52 / 6$ \\
EF (\%) & $35 \pm 11$ \\
Post MI, n (\%) & $26(45.8 \%)$ \\
DCM, n (\%) & $16(27.1 \%)$ \\
Other, n (\%) & $16(27.1 \%)$ \\
1st procedure, n (\%) & $34(57.6 \%)$ \\
ICD/CRTD, n (\%) & $46(78 \%)$ \\
Electrical storm, n (\%) & $10(16.9 \%)$ \\
\hline
\end{tabular}

CRTD, cardiac resynchronization therapy defibrillator; DCM, dilated cardiomyopathy; EF, ejection fraction; ICD, implantable cardioverter defibrillator; MI, myocardial infarction

Table 2. Periprocedural characteristics of catheter ablation for ventricular tachycardia

\begin{tabular}{ll}
\hline & $\mathrm{N}=58$ \\
\hline 3D mapping system, n (\%) & $58(100 \%)$ \\
Epicardial approach, n (\%) & $7(12.1 \%)$ \\
Irrigated catheter, n (\%) & $58(100 \%)$ \\
Force-sensing catheter, n (\%) & $26(44.8 \%)$ \\
Multipolar mapping catheter, n (\%) & $40(69.0 \%)$ \\
Intracardiac echocardiography, n (\%) & $37(64.0 \%)$ \\
Pre-VT inducibility, n (\%) & $31(53.4 \%)$ \\
Total RF duration, median, (25\%-75\% [min]) & $14(6.5-26)$ \\
Procedure time (min) & $266 \pm 97$ \\
\hline
\end{tabular}

$\mathrm{RF}$, radiofrequency; VT, ventricular tachycardia 
Table 3. Causes of avoiding ventricular tachycardia induction after ablation

\begin{tabular}{lll}
\hline & $\mathrm{N}=58$ & Suspension before sufficient RF delivery \\
\hline Non-inducibility before ablation, $\mathrm{n}(\%)$ & $27(46.6 \%)$ & $0(0 \%: 0 / 27)$ \\
Long procedure time $(>6 \mathrm{hr}), \mathrm{n}(\%)$ & $16(25.9 \%)$ & $1(6.3 \%: 1 / 16)$ \\
Complications, n $(\%)$ & $6(10.3 \%)$ & $5(83.3 \%: 5 / 6)$ \\
Intolerant hemodynamic state, $\mathrm{n}(\%)$ & $6(10.3 \%)$ & $2(33.3 \%: 2 / 6)$ \\
Inaccessible or unsafe target, n $(\%)$ & $3(5.2 \%)$ & $3(100 \%: 3 / 3)$ \\
\hline
\end{tabular}

$\mathrm{RF}$, radiofrequency.

\section{Figure legends}

Figure 1: Kaplan-Meier curves of recurrent ventricular tachycardia after ablation procedures where VT was not inducible before radiofrequency ablation and the rest of procedures (others) during the follow-up period.

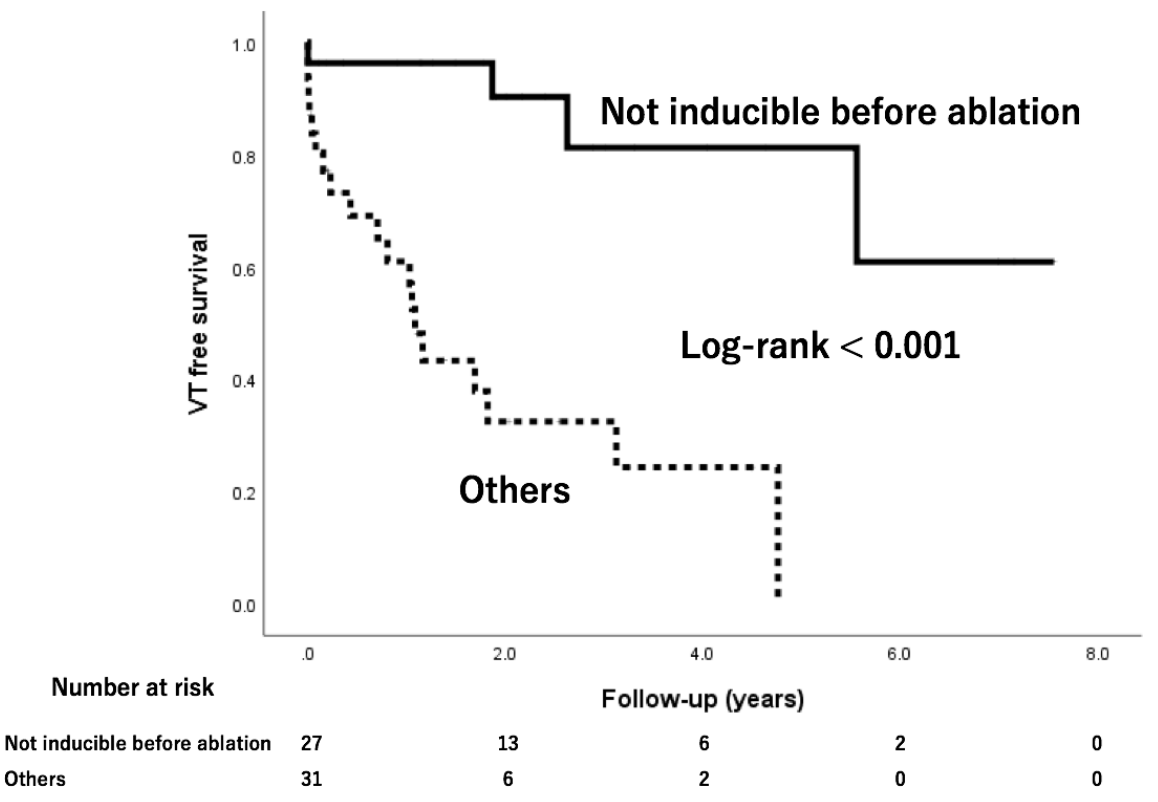

VT, ventricular tachycardia.

Supplementary Figure 1 : Kaplan-Meier curves of recurrent ventricular tachycardia after ablation procedures among four groups during the follow-up period.

VT, ventricular tachycardia; PES, program electrical stimulation; NA, not assessed. 


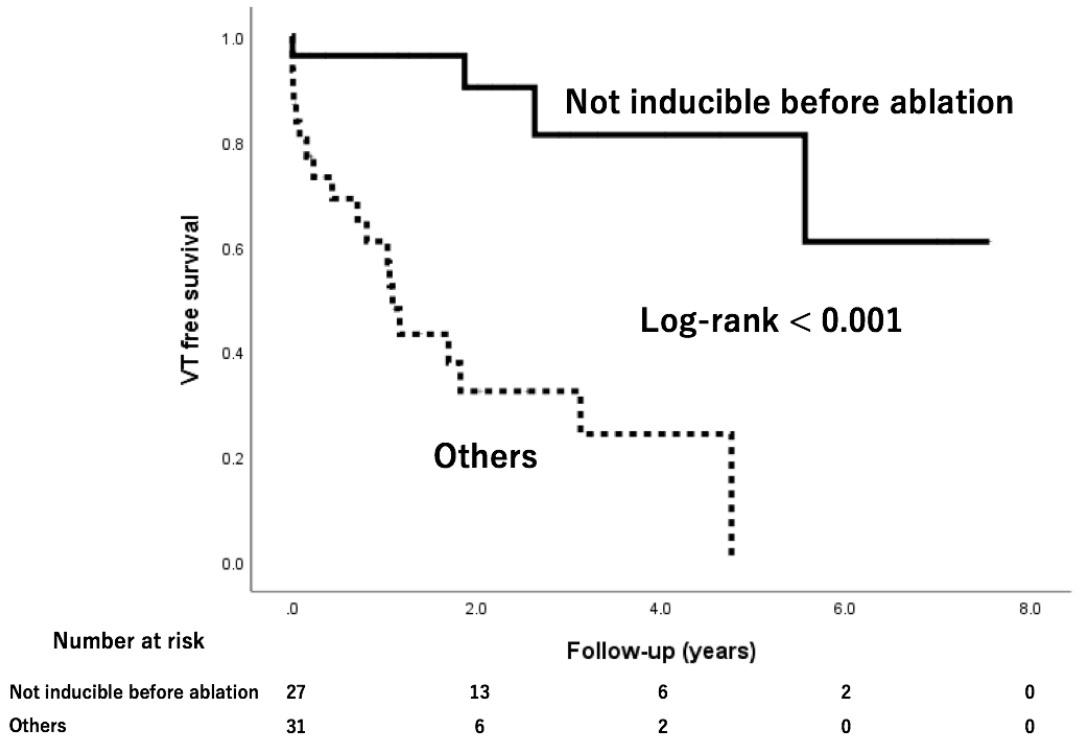

